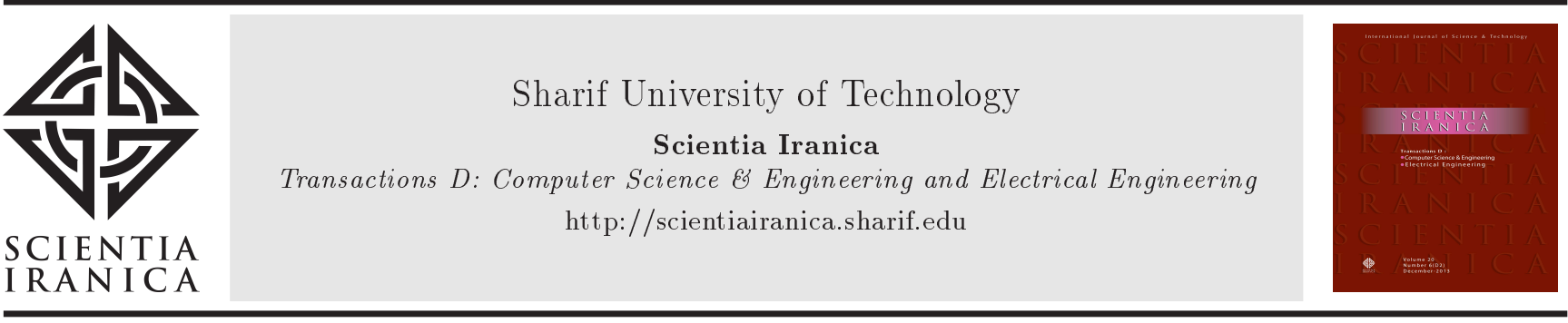

\title{
An experimental study regarding economic load dispatch using search group optimization
}

\author{
K. Bhattacharjee ${ }^{\mathrm{a}, *}$ and N. Patel \\ Institute of Technology, Department of Electrical Engineering, Nirma University, SG Highway, Gota, Ahmedabad-382481, India.
}

Received 17 September 2018; received in revised form 17 December 2018; accepted 23 February 2019

\author{
KEYWORDS \\ Economic load \\ dispatch; \\ Optimization; \\ Prohibited operating \\ zone; \\ Search group \\ optimization; \\ Valve-point loading.
}

\begin{abstract}
Power system network is formed to mainly generate power through all generators to fulfil total load demand and compensate for transmission line losses. The Economic Load Dispatch (ELD) problem is considered one of the most important problems of cost minimization in power system operations. Various approaches have been employed so far to solve the ELD problem. In this paper, a powerful Search Group Optimization ( $\mathrm{SGO}$ ) technique was implemented to solve the ELD problem. SGO maintains a good balance between its exploitation and exploration phases. This optimization technique tends to find promising regions of the search space from the first iteration onwards. The algorithm uses five important steps to reach an optimal solution to the ELD problem and they include initial population, initial selection of search group, search group mutation, family generation, and new search group selection. Using these five steps, the SGO tends to make a smooth transition towards the optimized solution. The SGO was applied to five test systems and the final results were compared to those of various other recently developed optimization techniques. The results proved the robustness, feasibility, effectiveness, and efficiency of SGO in terms of computational time and proximity to global optimum solution.

(C) 2020 Sharif University of Technology. All rights reserved.
\end{abstract}

\section{Introduction}

The problem of Economic Load Dispatch (ELD) is the one of the most important problems in the field of power system optimization. The ELD problem is required to satisfy total load demand using all generators while meeting all their physical and operational constraints. This load demand needs to be satisfied in the most economical manner. The ELD ensures that the total cost of thermal generation is minimized and the reliability of the entire system is also maintained. However, it should be ensured that the equality and inequality constraints of each and every generator are satisfied.

*. Corresponding author. Tel.: 98 32832822

E-mail addresses: kuntal.bhattacharjee@nirmauni.ac.in (K. Bhattacharjee); 16meee18@nirmauni.ac.in (N. Patel)

doi: $10.24200 /$ sci. 2019.51798 .2367
Initially, there are many optimization techniques that were based on the classical approach and some of them include gradient method [1], Lagrangian relaxation [2], Quadratic Programming (QP) [3], Hopfield modeling framework [4], Linear Programming (LP) [5], and Dynamic Programming (DP) [6]. These methods assume a linear increasing cost function and have been used to solve the ELD problems.

The difficulty that puts these classical approaches at a disadvantage is that they tend to converge towards a local optimum in the search space and then, they get diverted from the global optimum solution. The main drawback of the DP approach is that it needs very large dimensions of data, hence requiring a huge amount of programming efforts. Because of the presence of non-linear equations such as ramp rate limit, non-smooth cost functions, and discontinues prohibited operating zones (POZs), classical techniques are not efficient enough to identify the global optimum 
solution. Because of the non-linear characteristic of the ELD problem, the optimization techniques having a classical approach tend to move towards a local optimum, hence avoiding the global optima. Therefore, it is very important to find an optimization technique that can not only overcome all the disadvantages of the classical techniques but also avoid the local optima and directly move towards the global optimum solution within the least computational time. There are many optimization techniques like hopfield neural network [7] that have been implemented to solve the ELD problem. However, the main disadvantage to artificial intelligence techniques is that they take a large number of iterations to reach the global optimum solution. Thus, they prove to be a bit slow in their approach to the global optimum solution. With the development of computer technology, many new populationbased heuristic optimization techniques have been introduced including Differential Evolution (DE) [8], Hybrid Evolutionary Programming (HEP) [9], Evolutionary Programming (EP) [10], Civilized Swarm Optimization (CSO) [11], Particle Swarm Optimization (PSO) [12], Craziness-based PSO (CRPSO) [13], Hybrid PSO (HPSO) [14], Modified PSO (MPSO) [15], Hybrid GA (HGA) [16], Genetic Algorithm (GA) [17], Adaptive Real Coded GA (ARCGA) [18], Bacteria Foraging Optimization (BFO) [19], Modified Artificial Bee Colony (ABC) [20], Modified Biogeography based Optimization (BBO) [21], Seeker Optimization Algorithm (SOA) [22], Ant Colony Optimization (ACO) [23], Tabu Search (TS) [24], Quasi Oppositional BBO (QOBBO) [25], Oppositional BBO (OBBO) [26], and Harmony Search Algorithm (HSA) [27] for solving ELD problems. Other optimization algorithms including Opposition-based Harmony Search Algorithm (OHSA) were proposed to solve the ELD problem in [28]. Krill Herd Algorithm (KHA) [29] was also successfully applied to solve the ELD problems. Teaching Learning-Based Optimization (TLBO) was implemented in $[30,31]$. This optimization technique divides search agents into teaching and learning phases and different subjects offered to the learners are considered design variables of the optimization problem. Modification of TLBO optimization algorithm called quasi TLBO was proposed in [32], which successfully solved the ELD problem. Oppositional Real Coded Chemical Reaction Optimization (ORCCRO) [33] is capable to solve non-linear and non-quadratic equations with smoother transition. Some new algorithms like backtracking search optimization [34], exchange market algorithm [35], enhanced PSO [36], evolutionary PSO [37], and ameliorated grey wolf optimization [38] have been introduced recently, in which the PSO and the grey wolf technique have been modified to solve the ELD problem. In the ameliorated grey wolf optimization, the conventional grey wolf algorithm has been modified by changing the base equation and focusing more on the exploration and exploitation phases of the algorithm. In the evolutionary PSO, the conventional PSO technique has been modified by using an evolutionary term, which helps evolve the fitness of each search in the subsequent iteration. Sine cosine algorithm [39] has been used to solve the ELD problem. This algorithm uses trigonometric functions to find the optimal results. In this technique, there are two equations that help search agents reach the optimized answer. However, many of the algorithms stated above face some difficulties in avoiding the local optima and thus, there is a need for finding a strong optimization technique.

Initially, the classical and derivative techniques were used to solve the problem of ELD; however, given that the problem gets more complex in nature, the classical techniques fail to give optimal results. Thus, soft computing techniques should be used to solve the problem of ELD. A powerful Search Group Optimization (SGO) technique was proposed in [40] and it was characterized by a more efficient exploration and exploitation ability and a shorter computational time than other optimization techniques. The SGO follows five steps to reach an optimal solution: initial population, initial selection of search group, search group mutation, family generation, and new search group selection. In this paper, the SGO is applied to five different types of test systems and the results obtained are compared to other optimization techniques. The main advantage of using the SGO technique is that with every iteration, the size of the search space keeps on decreasing. This reduction in the search space helps shorten the time required for reaching an optimal solution and it facilitates quick transition from the exploration to exploitation phase. Once the area of the optimal value is well defined, the exploitation process helps find the final solution to any problem.

Section 2 gives the problem formulation. Section 3 introduces a brief explanation of the SGO and the results. Section 4 gives the simulations. Finally, conclusion is drawn in Section 5.

\section{Problem formulation}

The problems of ELD are expressed as convex or non-convex problems with some linear and nonlinear constraints for different applications.

The objective function of ELD with quadratic cost function based on Eq. (1) is as follows [41]:

$$
F_{\text {Cost }}=\min \sum_{a=1}^{N}\left(\alpha_{a}+\beta_{a} P_{a}+\gamma_{a} P_{a}^{2}\right) .
$$

For realistic and practical application of ELD problem, the smooth quadratic cost function was modified by 
adding sinusoidal terms of ripples input-output curve with valve point effects. The valve point effect-based cost function of ELD is given below [41]:

$$
\begin{aligned}
F_{\text {Cost }}= & \min \sum_{a=1}^{N}\left(\alpha_{a}+\beta_{a} P_{a}+\gamma_{a} P_{a}^{2}+\mid \delta_{a}\right. \\
& \left.\times \sin \left\{\varepsilon_{a}\left(P_{a}^{\min }-P_{a}\right)\right\} \mid\right),
\end{aligned}
$$

where $\alpha_{a}, \beta_{a}, \gamma_{a}, \delta_{a}$, and $\varepsilon_{a}$ are the constant values of fuel cost function. $N$ is the total number of thermal generators. The power generated by each generator is $P_{a}$. Lower and higher limits of power generation are characterized by $P_{a}^{\min }$ and $P_{a}^{\max }$. Power generation by each unit is followed by generating capacity constraint:

$$
P_{a}^{\min } \leq P_{a} \leq P_{a}^{\max } .
$$

This is the inequality constraint of ELD problems. The equality constraint or real power balance constraint of ELD is based on Eq. (4):

$$
\sum_{a=1}^{N} P_{a}-P_{D}-P_{\text {Loss }}=0
$$

where $P_{D}$ is the total system active power demand and total transmission loss $P_{\text {Loss }}$ is calculated by using the $B$-matrix loss coefficients expressed as [41]:

$$
P_{\text {Loss }}=\sum_{a=1}^{N} \sum_{b=1}^{N} P_{a} B_{a b} P_{b}+\sum_{a=1}^{N} B_{0 a} P_{a}+B_{00}
$$

Ramp rate limit is another constraint considered in ELD problems to increase the life of generators. The active power of a particular generator needs to be generated within the given operating limits. Due to abrupt changes in power generation at any interval, heavy load is imposed on the generators which can be harmful; thus, changes in power generation should be limited to upper and lower values, which are considered as rate limits of upper ramp and down ramp. Therefore, based on the ramp rate limit, power generation by any unit should follow the equations below:

$$
\begin{array}{ll}
P_{a}-P_{a 0} \leq U R_{a} & \text { (as generation rises), } \\
P_{a 0}-P_{a} \leq D R_{a} & \text { (as generation declines), }
\end{array}
$$

and:

$$
\max \left(P_{a}^{\min }, P_{a 0}-D R_{a}\right) \leq \min \left(P_{a}^{\max }, P_{a 0}+U R_{a}\right),
$$

where $P_{a 0}$ is the power generation of the previous $a$ th interval; $U R_{a}$ and $D R_{a}$ are the up- and down-ramp limits.
Different faults in the machines, boilers, feed pumps, steam valve operation, and vibration in the bearing, etc., the constraints like POZ are considered in ELD problems. To protect the generator, it should not generate power in the prohibited zone. Mathematically, POZ can be expressed as follows:

$$
\left.\begin{array}{c}
P_{a}^{\min } \leq P_{a} \leq P_{a, 1}^{l} \\
P_{a, j-1}^{u} \leq P_{a} \leq P_{a, j}^{l} \\
P_{a, n}^{u} \leq P_{a} \leq P_{a}^{\max }
\end{array}\right\} ; \quad j=1 \quad \text { to } \quad n
$$

where $P_{a, j}^{u}$ and $P_{a, j}^{l}$ are the upper and lower limits of the $j$ th POZ of the $a$ th unit. Total number of POZ of the $a$ th unit is $n$.

For a system with $n$ number of generators and having $n_{F}$ fuel options for each unit, the entire cost function can be expressed as follows:

$$
\begin{aligned}
F_{i p}\left(P_{i}\right)= & a_{i p}+b_{i p} P_{i}+c_{i p} P_{i}^{2} \\
& +\left|e_{i p} \times \sin \left\{f_{i p} \times\left(P_{i p}^{\min }-P_{i}\right)\right\}\right|,
\end{aligned}
$$

where $p=1,2, \cdots, n_{F}$. Calculation of slack generator is one of the important parts of ELD problem formulations. If $N$ is the total number of generators, then the $(N-1)$ number of power generations should initially be calculated randomly based on Relations (3), (6)(9). The remaining generator (let $N$ th), called slack generator, must be calculated using Eq. (4). The value of slack generator is given below:

$$
\begin{aligned}
& P_{N}=P_{D}-\sum_{a=1}^{N-1} P_{a} \quad \text { (without transmission losses), } \\
& P_{N}=P_{D}+P_{\text {Loss }}-\sum_{a=1}^{N-1} P_{a} \quad \text { (with transmission losses). }
\end{aligned}
$$

Transmission loss $\left(P_{\text {Loss }}\right)$ is also related to power generations based on Eq. (5); therefore, Eq. (11) is further modified and given below:

$$
\begin{aligned}
B_{N N} P_{N}^{2} & +P_{N}\left(2 \sum_{a=1}^{N-1} B_{N a} P_{a}+\sum_{a==1}^{N-1} B_{0 N}-1\right) \\
& +\left(P_{D}+\sum_{a=1}^{N-1} \sum_{b=1}^{N-1} P_{a} B_{a b} P_{b}\right. \\
& \left.+\sum_{a=1}^{N-1} B_{0 a} P_{a}-\sum_{a=1}^{N-1} P_{a}+B_{00}\right)=0
\end{aligned}
$$

\section{Search Group Optimization (SGO)}

In order to find a solution closer to the optimized solution, a powerful SGO is proposed that has a good 
balance between exploration and exploitation phases of the algorithm. The basic idea of the SGO is to find the promising areas of the search space in the first iteration itself. The SGO finds a solution nearest to the global solution and then, compares it to the solution obtained in the previous iterations. If the tolerance is within the permissible limits, then the answer is accepted; if not, then the iterations continue. There are five steps that make up this algorithm. They are stated in detail as follows:

\subsection{Initial population}

An initial population set $P$ is generated from the search domain.

$$
\begin{aligned}
P_{i j} & =x_{j}^{\min }+\left(x_{j}^{\max }-x_{j}^{\min }\right) * U \quad j=1,2, \cdots, n_{D} \\
i & =1,2, \cdots, n_{\text {pop }}
\end{aligned}
$$

where $P_{i j}$ is the $j$ th design variable belonging to the $i$ th individual of the population set $P$. $U[0,1]$ is a randomly generated variable ranging from 0 to $1 . x_{j}^{\min }$ and $x_{j}^{\max }$ are the lower and upper bounds of the $j$ th design variable. $n_{D}$ is the total number of design variables. $n_{\text {pop }}$ is the total size of the population. This process initializes the total number of search agents that will be used to screen the entire search space. Once the total size of the population set $P$ is decided, then the selected search agents having higher fitness than other search agents are selected to move towards the optimum solution.

\subsection{Initial selection of search group}

In the previous stage, the population set $P$ is created and it has all the elements placed randomly based on their objective function. Then, each set of the population is sorted rank wise by comparing their fitness functions. The elements having the highest fitness are placed in the first row and the elements having the lowest fitness are placed in the last row. A matrix $R$ is created, in which each row represents a set of individuals. The $j$ th row represents the $i$ th member of the search group.

\subsection{Search group mutation}

So as to maximize the ability to search, the search group $R$ is mutated in each and every iteration. In this process, the individuals in the search group $R$ are replaced by new individuals generated based on Eq. (15). The main advantage of this mutation process is that the areas of the search space away from the current search group $R$ 's position are properly explored. Hence, the entire search space gets properly explored. The new individuals are generated with the equation:

$$
x_{j}^{m u t}=E\left[R_{j}\right]+t \varepsilon \sigma\left[R_{j}\right] \quad \text { for } \quad j=1,2, \ldots, n_{D} .
$$

Here, $x_{j}^{\text {mut }}$ is the $j$ th variable of the given mutated individual. $E$ is the mean value and $\sigma$ is the standard deviation operator. Different values of the variable $\varepsilon$ at different times in Eq. (15) are selected randomly within $[-1,1]$. Variable $t$ from Eq. (15) is used for controlling the location of the newly generated individual in the search space and the numerical value can be taken within 1,2 , or $3 . R_{j}$ is the $j$ th column in the search group matrix. After mutation operation, the elements are changed entirely and a new matrix $R_{\text {new }}$ is created which comprises all the mutated elements. Each set of individuals for $R$ and $R_{n e w}$ is compared based on their fitness. The suitable set of individuals (based on their fitness) is replaced and assigned to matrix $S$. Thus, a new search group matrix $S$ is created.

\subsection{Family generation}

In this process, a new family is created by the members of the search group and this set is denoted by $F$. Thus, once the entire search group is finalized, each member will generate a family by the equation:

$$
x_{j}^{\text {new }}=R_{i, j}+\alpha \varepsilon \quad \text { for } \quad j=1,2, \ldots, n_{D},
$$

where $\alpha$ is the control parameter for the size of perturbation. As the iteration count increases, the size of $\alpha$ keeps on decreasing. The updated parameters are given by:

$$
\alpha^{k+1}=b \alpha^{k}
$$

where $b$ is a parameter of the SGO and can be assigned using Eq. (18):

$$
b=\max \left(1-\frac{4 \times k_{\text {current }}}{k_{\max }}, 0.25-\frac{k_{\text {current }}}{k_{\max }}\right),
$$

where $k_{\text {current }}$ and $k_{\max }$ are the current and maximum numbers of iterations, respectively. $\alpha^{k}$ is the value of $\alpha$ in the $k^{t h}$ iteration. The parameter $\alpha^{k}$ facilitates the exploration phase of the algorithm. Initially, $\alpha$ has a large value, which means that it allows the individuals to move freely in the search space and explore the entire search space. However, as the iterations progress, the value of $\alpha$ decreases, thus restricting the movement space for the individuals. Thus, the individuals stay in the neighbourhood only. Hence, initially, the value of $\alpha$ should be large enough to allow the individuals to freely move in the search space and find the promising regions of the entire search space. The main advantage of this process is that the higher the fitness of a search agent, the larger the number of individuals that it will generate.

\subsection{New search group selection}

In this phase, when the number of iterations exceeds a predefined value, then the exploration phase of the algorithm will end. Now, the new search group will start exploiting the present search area. This phase 
is called the local phase, given that in this phase, the individuals do not go to the global regions to explore the search space; however, they remain in the current search space to find an optimized solution.

There are a few features unique to SGO that make this algorithm different from other optimization techniques: (1) the higher the fitness of the group, the larger the number of individuals that it will generate; (2) the implementation of the standard deviation and the mean value in the mutation process; (3) the presence of the global and local phases that allow smooth transition from the exploration to the exploitation phase.

\section{Algorithm for $S G O$}

- Initially, the total number of search agents is assigned. Each of the elements of the search agents should maintain their upper and lower limits. These search agents have the duty to screen the entire search space and find the optimum solution. The total number of search agents is decided depending on the number of variables present in the objective function of the problem. This initialization was presented in Eq. (14);

- The initial population set is created by assigning the values of search agents. This set is denoted by $P$;

- In the subsequent step, the objective function of each and every individual is calculated;

- A new search group $R$ is created which comprises all those search agents characterized by a higher fitness function. The search agents with a high fitness function are only allowed to stay in the search group $R$, which is having only half the population of the set $P$;

- Now, to maximize the search ability of the search agents, each and every element in the search group $R$ is mutated. In this process, the location of a search agent is given in binary integer number. Any binary number represents a combination between 0 and 1. The number of ones and zeros of the location of each search agents is complimented. Thus, the new search agent produced is present on the entire opposite side of the search space. These search agents are created using Eq. (15). This allows the algorithm to select only those search agents with high fitness only;

- If the mutated search agent has higher fitness than the original search agent, then the original search agent is replaced;

- Now, the new set that comprises the mutated and original search agents is created. This new set will be used for screening the search space. This set is denoted by $F$ and is created using Eq. (16);
- Change in the value of $\alpha$ (input equation number) facilitates a smooth transition from the exploration to exploitation phase. When the value of $\alpha$ is large, then the exploration of the search space takes place; however, given that the iterations will pass, the value of $\alpha$ decreases and then, the exploitation phase begins. The updating of $\alpha$ is done using Eq. (17);

- When a predefined value of iterations is exceeded, the algorithm reaches its final value;

- Now, the search agents are completely in the exploitation phase and will not go to any other search space to explore the possibility of an optimum solution.

The flowchart of SGO algorithm is given in Figure 1.

\subsection{Consecutive steps of $S G O$ algorithm integrated into the ELD problem}

In this subsection, the steps to solve the ELD problem by the implementation of SGO are explained. The detailed sequential steps for solving the ELD problem are explained below:

i. Initialization of various parameters takes place in the first step. Various variables including the lower bound, upper bound, total power demand $P_{D}$, etc. are initialized. The total number of generators and total number of search agents are denoted by $m$ and Popsize, respectively; The search agent matrix is represented as follows:

$$
X=X_{i}=\left[X_{1}, X_{2}, X_{3}, \cdots, X_{\text {Popsize }}\right] \text {, }
$$

where $i=1,2,3, \ldots$, Popsize. For ELD problem, the search agent matrix is assigned as active power generation and is represented as follows:

$$
\begin{aligned}
{\left[X_{i j}\right]=} & {\left[X_{i 1}, X_{i 2}, X_{i 3}, \cdots, X_{i m}\right]=\left[P_{i 1},\right.} \\
& \left.P_{i 2}, P_{i 3}, \cdots, P_{i m}\right]=\left[P_{i j}\right],
\end{aligned}
$$

where $m=$ number of generators.

ii. Each of the elements of the search agent should follow Relations (3), and (6)-(9). If various effects including ramp rate limit and the POZ are considered, then the equation should be satisfied based on Eqs. (6), (7), (8), and (9), respectively.

iii. For ELD problem, the objective function considers the fuel cost of power generation and can use (1) when quadratic fuel cost function is applied and also use (2) when valve point loading effect is considered. This objective function serves as the base of the algorithm. This function needs to minimize the cost of the power generation in the system. The objective function of fuel cost is calculated based on the power generation $\left(P_{i j}\right)$ from step i. 


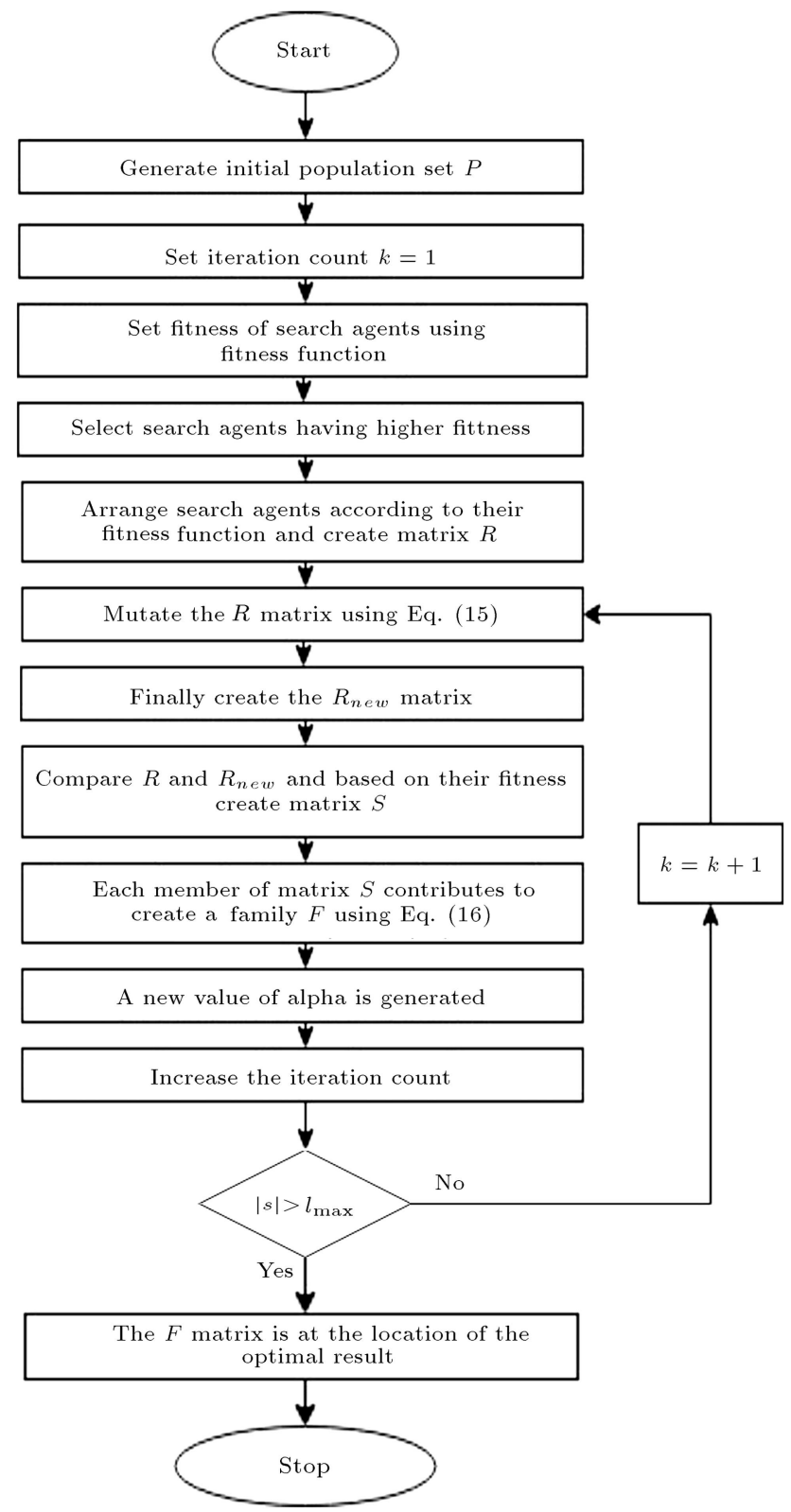

Figure 1. Flowchart of Search Group Optimization (SGO) algorithm.

iv. The main working mechanisms of the algorithm begin here. A new search group $R$ is formed which comprises all those search agents with a higher fitness function. The search agents with a high fitness function are only allowed to stay in the search group $R$, which is having only half the population of the set $P$.

v. Now, to maximize the search ability of the search agents, each and every element in the search group $R$ is mutated. In this process, the location of a search agent is given in the binary integer number. Any binary number represents a combination between 0 and 1 . The number of ones and zeros of the location of each search agents is complimented.
Thus, the new search agent produced is present on the entire opposite side of the search space. This allows the algorithm to select only those search agents that are characterized by high fitness only. If the mutated search agent has higher fitness than the original search agent, then the original search agent is replaced.

vi. Change in the value of $\alpha$ (input equation number) facilitates a smooth transition from the exploration to exploitation phase. When the value of $\alpha$ is large, then the exploration of the search space takes place; however, as the iterations pass, the value of $\alpha$ decreases and then, the exploitation phase will begin.

\section{Results and simulations}

To prove the effectiveness and efficiency of the SGO, five sets of cases with varying degrees of complexity were considered and the final results were compared to those of different other existing optimization methods. The program was written using MATLAB-2017A programming tool and executed by a $1.7 \mathrm{GHz}$ Intel core i7 personal computer with 8-GB RAM.

Test Case 1: Six generator units were considered in Test Case 1, where transmission losses were taken into account. The total power demand was $1263 \mathrm{MW}$. The input data were taken from [42] and the system ran up to 400 iterations. The number of search agents used was 50 in this case. In Test Case 1, the results of the SGO algorithm were compared with those of TLBO [42], CTLBO [42], and AIS [42] optimization techniques. According to the graph and the table, the minimum cost was first reached by using the SGO algorithm and the rest of the optimization techniques took a very short amount of time. In Table 1 , the minimum fuel cost for 6 generator units was $15377.8907 \$ / \mathrm{hr}$. and it could be determined by the proposed algorithm, which outperformed TLBO [42], CTLBO [42], and AIS [42]. The minimum, maximum, and average fuel costs obtained after 50 trials are presented in Table 2. The convergence characteristics of SGO are shown in Figure 2;

Test Case 2: Ten generator units were considered in Test Case 2, in which transmission losses were neglected. This test case considered multi-fuel cost and valve-point loading effect. The total power demand was $2700 \mathrm{MW}$. The input data were taken from [34]. The number of search agents used was 50 in this case. In this test case, the results obtained using SGO algorithm were compared with those of PSO-LRS [43], APSO [43], and CBPSO-RVM [43] optimization techniques to prove the effectiveness of SGO algorithm. According to Table 3, the minimum cost was first 
Table 1. Comparison between Search Group Optimization (SGO) and other techniques for the 6-unit test system in terms of optimum power output and fuel cost.

\begin{tabular}{ccccc}
\hline \multirow{2}{*}{ Unit } & \multicolumn{4}{c}{ Power output (MW) } \\
\cline { 2 - 5 } & SGO & TLBO [42] & CTLBO [42] & AIS [42] \\
\cline { 2 - 5 } P1 & 436.4684 & 446.7270 & 449.4980 & 458.2904 \\
P2 & 166.8021 & 173.4890 & 173.4810 & 168.0518 \\
P3 & 249.3160 & 173.4890 & 264.9700 & 262.5175 \\
P4 & 125.8384 & 138.8320 & 127.4610 & 139.0604 \\
P5 & 190.8636 & 165.6500 & 173.8420 & 178.3936 \\
P6 & 100.8060 & 86.9460 & 86.2390 & 69.3416 \\
Transmission loss (MW) & 7.1800 & 12.4180 & 12.4900 & 13.1997 \\
Power generated (MW) & 1270.0945 & 1275.4180 & 1275.4900 & 1275.6550 \\
Fuel cost (\$/hr) & $\mathbf{1 5 3 7 7 . 8 9 0 7}$ & $15,442.5200$ & $15,441.6970$ & $15,448.0000$ \\
\hline
\end{tabular}

Table 2. Minimum maximum and average cost obtained by Search Group Optimization (SGO) and various optimization techniques for 6 generator units (50 trials).

\begin{tabular}{|c|c|c|c|c|c|}
\hline \multirow{2}{*}{ Methods } & \multicolumn{3}{|c|}{ Generation cost $(\$ / \mathrm{hr})$} & \multirow{2}{*}{$\begin{array}{c}\text { Time/ } \\
\text { iteration (S) }\end{array}$} & \multirow{2}{*}{$\begin{array}{l}\text { No. of hits to } \\
\text { minimum solution }\end{array}$} \\
\hline & Maximum & Minimum & Average & & \\
\hline SGO & 15378.2541 & 15377.8907 & 15377.8907 & 0.45 & 50 \\
\hline TLBO [42] & 15450.3685 & $15,442.5200$ & 15445.8163 & 0.98 & 29 \\
\hline CTLBO [42] & 15449.0236 & $15,441.6970$ & 15445.9464 & $\mathrm{NA}^{*}$ & 21 \\
\hline AIS [42] & NA & 623.9588 & NA & NA & NA \\
\hline
\end{tabular}

*NA: Not Available

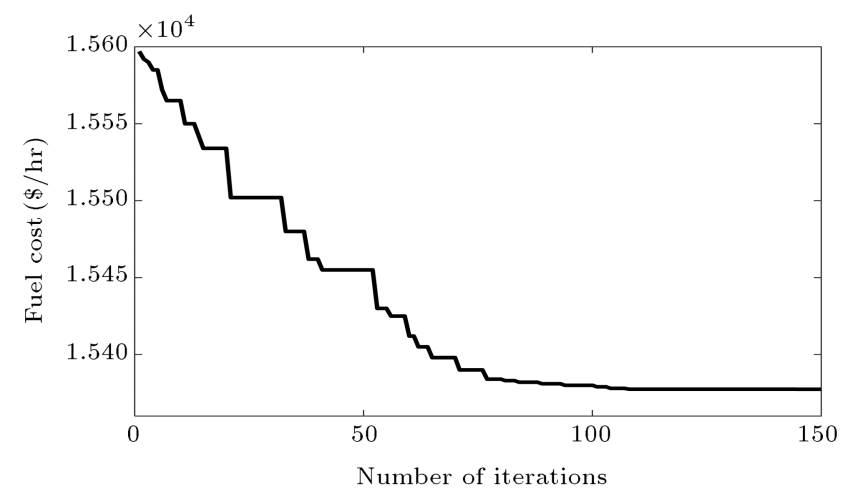

Figure 2. Convergence characteristics of Search Group Optimization (SGO) for 6 generator units.

reached by using the SGO algorithm and the rest of the optimization techniques took a minimum amount of time, compared to other. In Table 3 , the minimum fuel cost for 10 generator units was $623.9170 \$ / \mathrm{hr}$ and it was obtained by the proposed algorithm, which outperformed PSO-LRS [43], APSO [43], and CBPSORVM [43]. The minimum, maximum, and average fuel costs obtained after 50 trials are presented in Table 4 . The convergence characteristics of SGO are shown in Figure 3;

Test Case 3: In this system, 15 units of generators were considered and transmission loss was considered. The total load demand was 2630 MW. The minimum fuel cost was calculated using SGO. The input data were taken from [35] and the system ran for 500 iterations. The POZ and ramp rate limit were considered in this case. In Test Case 3, the results of the SGO algorithm were compared with those of Exponential Moving Average (EMA) [35] and GAAPI [35] optimization techniques. According to Table 5 and Figure 4, 
Table 3. Comparison between Search Group Optimization (SGO) and other techniques for the 10-unit test system in terms of optimum power output and fuel cost.

\begin{tabular}{ccccccccc}
\hline & \multicolumn{7}{c}{ Power Output (MW) } \\
\cline { 2 - 8 } Unit & $\begin{array}{c}\text { Fuel } \\
\text { type }\end{array}$ & SGO & $\begin{array}{c}\text { Fuel } \\
\text { type }\end{array}$ & $\begin{array}{c}\text { PSO-LRS } \\
{[\mathbf{4 3}]}\end{array}$ & $\begin{array}{c}\text { Fuel } \\
\text { type }\end{array}$ & $\begin{array}{c}\text { APSO } \\
{[\mathbf{4 3}]}\end{array}$ & $\begin{array}{c}\text { Fuel } \\
\text { type }\end{array}$ & $\begin{array}{c}\text { CBPSO-RVM } \\
{[\mathbf{4 3}]}\end{array}$ \\
\hline P1 & 2 & 217.0407 & 2 & 219.0155 & 2 & 223.3377 & 2 & 219.2073 \\
P2 & 1 & 211.8944 & 1 & 213.8901 & 1 & 212.1547 & 1 & 210.2203 \\
P3 & 1 & 281.6792 & 1 & 283.7616 & 1 & 276.2203 & 1 & 278.5456 \\
P4 & 3 & 238.2056 & 3 & 237.2687 & 3 & 239.4176 & 3 & 239.3704 \\
P5 & 1 & 279.8321 & 1 & 286.0163 & 1 & 274.6411 & 1 & 276.412 \\
P6 & 3 & 239.2547 & 3 & 239.3987 & 3 & 239.7953 & 3 & 240.5797 \\
P7 & 1 & 290.2798 & 1 & 291.1767 & 1 & 285.5406 & 1 & 292.3267 \\
P8 & 3 & 240.2228 & 3 & 241.4398 & 3 & 240.6270 & 3 & 237.7557 \\
P9 & 3 & 425.5958 & 3 & 416.9721 & 3 & 429.3104 & 3 & 429.4008 \\
P10 & 1 & 275.9942 & 1 & 271.0623 & 1 & 278.9553 & 1 & 276.1815 \\
Fuel cost $(\$ / \mathrm{hr})$ & - & $\mathbf{6 2 3 . 9 1 7 0}$ & - & 624.2297 & - & 624.0145 & - & 623.9588 \\
\hline
\end{tabular}

Table 4. Minimum, maximum, and average costs obtained by Search Group Optimization (SGO) and various optimization techniques for 10 generator units (50 trials).

\begin{tabular}{|c|c|c|c|c|c|}
\hline \multirow{2}{*}{ Methods } & \multicolumn{3}{|c|}{ Generation cost $(\$ / \mathrm{hr})}$. & \multirow{2}{*}{$\begin{array}{c}\text { Time/ } \\
\text { iteration (S) }\end{array}$} & \multirow{2}{*}{$\begin{array}{l}\text { No. of hits to } \\
\text { minimum solution }\end{array}$} \\
\hline & Maximum & Minimum & Average & & \\
\hline SGO & 625.5478 & 623.9170 & 623.9170 & 0.51 & 50 \\
\hline PSO-LRS [43] & 626.7210 & 624.2297 & 625.3756 & 0.98 & 27 \\
\hline APSO [43] & 628.3947 & 624.0145 & 626.5550 & $\mathrm{NA}^{*}$ & 21 \\
\hline CBPSO-RVM [43] & NA & 623.9588 & NA & NA & NA \\
\hline
\end{tabular}

*NA: Not Available.

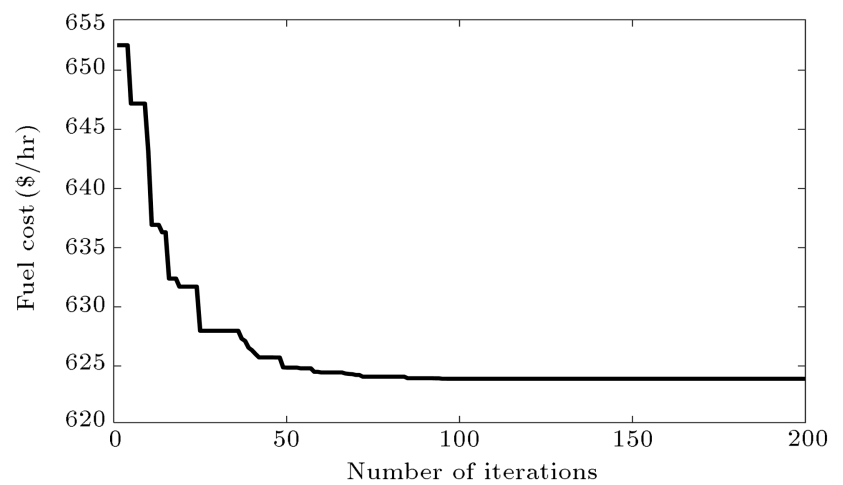

Figure 3. Convergence characteristics of Search Group Optimization (SGO) for 10 generator units.

the minimum cost was first reached by using the SGO algorithm and the rest of the optimization techniques took a minimum amount of time, compared to other. In Table 5, the minimum fuel cost for 15 generator units was $32697.2819 \$ / \mathrm{hr}$. and it was obtained by the

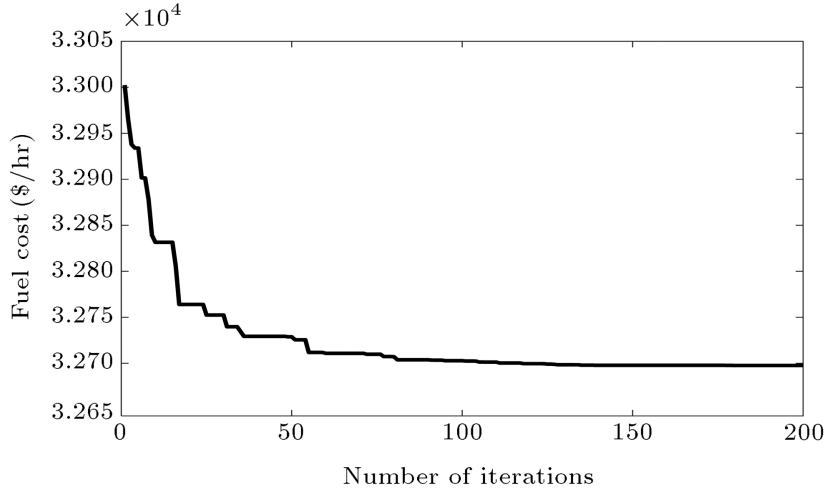

Figure 4. Convergence characteristics of Search Group Optimization (SGO) for 15 generator units.

proposed algorithm, which outperformed EMA [35] and GAAPI [35]. The minimum, maximum, and average fuel costs obtained after 50 trials are presented in Table 6. The convergence characteristics of SGO are given in Figure 4; 
Table 5. Comparison between Search Group Optimization (SGO) and other techniques for the 15-unit test system in terms of optimum power output and fuel cost.

\begin{tabular}{|c|c|c|c|}
\hline \multirow{2}{*}{ Unit } & \multicolumn{3}{|c|}{ Power output (MW) } \\
\hline & SGO & EMA [35] & GAAPI [35] \\
\hline $\mathrm{P} 1$ & 455.0000 & 455.0000 & 454.70 \\
\hline $\mathrm{P} 2$ & 380.0000 & 380.0000 & 380.00 \\
\hline P3 & 130.0000 & 130.0000 & 130.00 \\
\hline $\mathrm{P} 4$ & 130.0000 & 130.0000 & 129.53 \\
\hline $\mathrm{P} 5$ & 170.0000 & 170.0000 & 170.00 \\
\hline $\mathrm{P} 6$ & 460.0000 & 460.0000 & 460.00 \\
\hline $\mathrm{P} 7$ & 430.0000 & 430.0000 & 429.71 \\
\hline P8 & 71.4289 & 72.0415 & 75.35 \\
\hline P9 & 58.5965 & 58.6212 & 34.96 \\
\hline $\mathrm{P} 10$ & 160.0000 & 160.0000 & 160.00 \\
\hline P11 & 80.0000 & 80.0000 & 79.75 \\
\hline $\mathrm{P} 12$ & 80.0000 & 80.0000 & 80.00 \\
\hline $\mathrm{P} 13$ & 25.0000 & 25.0000 & 34.21 \\
\hline P14 & 15.0000 & 15.0000 & 21.14 \\
\hline P15 & 15.0000 & 15.0000 & 21.02 \\
\hline Transmission loss $(\$ / \mathrm{hr})$ & 29.9915 & 30.6626 & 30.3615 \\
\hline Power generation (MW) & 2659.6254 & 2660.6626 & 2660.3621 \\
\hline Fuel cost $(\$ / \mathrm{hr})$ & 32697.2819 & 32704.4503 & 32732.9515 \\
\hline
\end{tabular}

Table 6. Minimum, maximum, and average costs obtained by Search Group Optimization (SGO) and various optimization techniques for 15 generator units (50 trials).

\begin{tabular}{cccccc}
\hline \multirow{2}{*}{ Methods } & \multicolumn{3}{c}{ Generation cost $(\$ / \mathbf{h r})$} & $\begin{array}{c}\text { Time/iteration } \\
(\mathbf{s})\end{array}$ & $\begin{array}{c}\text { No. of hits to } \\
\text { minimum solution }\end{array}$ \\
\cline { 2 - 4 } & Maximum & Minimum & Average & $\mathbf{0 . 7 5}$ & $\mathbf{4 7}$ \\
\hline SGO & $\mathbf{3 2 6 9 8 . 1 5 7 4}$ & $\mathbf{3 2 6 9 7 . 2 8 1 9}$ & $\mathbf{3 2 6 9 7 . 3 3 4 4}$ & $\mathbf{0 . 8}$ & 24 \\
EMA [35] & 32708.3201 & 32704.4503 & 32706.4626 & 0.88 & 17 \\
GAAPI [35] & 32739.0147 & 32732.95 & 32736.9527 & $\mathrm{NA}^{*}$ & \\
\hline
\end{tabular}

* NA: Not Available.

Test Case 4: In this case, 38 generator units were considered and their transmission losses were neglected. The total power demand was $6000 \mathrm{MW}$. The input data were taken from [44] and the system ran for 400 iterations. In Test Case 4, the results of the SGO algorithm were compared with those of RCCRO [45], DE/BBO [45], and AGWO [38] optimization techniques. According to the graph and the table, the minimum cost was first reached by using the SGO algorithm and the rest of the optimization techniques took a minimum amount of time, as compared to other. In Table 7, the minimum fuel cost for 38 generator units was $9412256.3019 \$ / \mathrm{hr}$. and it was obtained by the proposed algorithm, which outperformed RCCRO [45], DE/BBO [45], and AGWO [38]. The minimum, maximum, and average fuel costs obtained after 50 trials are 
Table 7. Comparison between Search Group Optimization (SGO) and other techniques for the 38-unit test system in terms of optimum power output and fuel cost.

\begin{tabular}{|c|c|c|c|}
\hline \multirow[t]{2}{*}{ Unit } & \multicolumn{3}{|c|}{ Power output (MW) } \\
\hline & SGO & $\begin{array}{c}\text { RCCRO } \\
{[45]}\end{array}$ & $\begin{array}{c}\mathrm{DE} / \mathrm{BBO} \\
{[45]}\end{array}$ \\
\hline $\mathrm{P} 1$ & 426.1262 & 426.0061 & 426.6060 \\
\hline $\mathrm{P} 2$ & 430.7339 & 435.0539 & 426.6060 \\
\hline P3 & 421.9806 & 422.4470 & 429.6631 \\
\hline $\mathrm{P} 4$ & 438.6962 & 427.9425 & 429.6631 \\
\hline $\mathrm{P} 5$ & 425.4791 & 432.5756 & 429.6631 \\
\hline $\mathrm{P} 6$ & 435.1896 & 422.6355 & 429.6631 \\
\hline $\mathrm{P} 7$ & 440.6818 & 431.1825 & 429.6631 \\
\hline P8 & 420.8413 & 418.6959 & 429.6631 \\
\hline $\mathrm{P} 9$ & 114.5062 & 115.0654 & 114.0000 \\
\hline P10 & 114.4242 & 114.0000 & 114.0000 \\
\hline P11 & 118.3628 & 122.1920 & 119.7680 \\
\hline $\mathrm{P} 12$ & 123.5941 & 131.5714 & 127.0728 \\
\hline $\mathrm{P} 13$ & 110.0026 & 110.0000 & 110.0000 \\
\hline $\mathrm{P} 14$ & 90.0000 & 90.0000 & 90.0000 \\
\hline $\mathrm{P} 15$ & 82.0000 & 82.0059 & 82.0000 \\
\hline P16 & 120.0000 & 120.1242 & 120.0000 \\
\hline $\mathrm{P} 17$ & 161.7287 & 161.7578 & 159.5980 \\
\hline $\mathrm{P} 18$ & 65.0040 & 65.0000 & 65.0000 \\
\hline P19 & 65.0000 & 65.0000 & 65.0000 \\
\hline P20 & 271.9663 & 271.9461 & 272.0000 \\
\hline P21 & 271.7233 & 271.4465 & 272.0000 \\
\hline $\mathrm{P} 22$ & 259.4784 & 258.5583 & 260.0000 \\
\hline $\mathrm{P} 23$ & 127.8823 & 135.5357 & 130.6486 \\
\hline P24 & 10.0497 & 10.0000 & 10.0000 \\
\hline P25 & 116.5059 & 115.0635 & 113.3050 \\
\hline P26 & 84.7331 & 83.9506 & 88.0669 \\
\hline P27 & 35.3114 & 39.6814 & 37.5051 \\
\hline P28 & 20.0014 & 20.0000 & 20.0000 \\
\hline P29 & 20.0023 & 20.0000 & 20.0000 \\
\hline P30 & 20.0000 & 20.0055 & 20.0000 \\
\hline P31 & 20.0000 & 20.0003 & 20.0000 \\
\hline P32 & 20.0200 & 20.0000 & 20.0000 \\
\hline P33 & 25.0003 & 25.0002 & 25.0000 \\
\hline P34 & 18.000 & 18.0000 & 18.0000 \\
\hline P35 & 8.0005 & 8.0000 & 8.0000 \\
\hline P36 & 25.0000 & 25.0000 & 25.0000 \\
\hline P37 & 21.5802 & 23.7275 & 21.7820 \\
\hline P38 & 20.3922 & 20.8276 & 21.0621 \\
\hline $\begin{array}{c}\text { Fuel cost } \\
\qquad(\$ / \mathbf{h r})\end{array}$ & 9412256.3019 & 9412404.2774 & 9417235.7863 \\
\hline
\end{tabular}

presented in Table 8. The convergence characteristics of SGO are shown in Figure 5;

Test Case 5: In this case, 40 generator units were considered with their transmission losses neglected. The input data were taken from [46]. The total load demand was $10500 \mathrm{MW}$. In Test Case 5, the results of the SGO algorithm were compared with those of EMA [46] and Quantum behaved Particle Swarm Algorithm (QPSO) [46] optimization techniques. According to the graph and the table, the minimum cost was first reached by using the SGO algorithm and the rest of the optimization techniques took a minimum amount of time, compared to other. In Table 9 , the minimum fuel cost for 40 generator units was $121412.5347 \$ / \mathrm{hr}$. and it was obtained by the proposed algorithm, which outperformed EMA [46] and QPSO [46]. The minimum, maximum, and average fuel costs obtained after 50 trials are presented in Table 10 . The convergence characteristics of SGO are shown in Figure 6.

\subsection{Tuning of parameters for the $S G O$}

To obtain an optimized solution using SGO, it is imperative to obtain proper values of parameters $\alpha$, $\varepsilon$, and $t$. Tuning of these parameters is very important for obtaining an optimized solution. Different values of

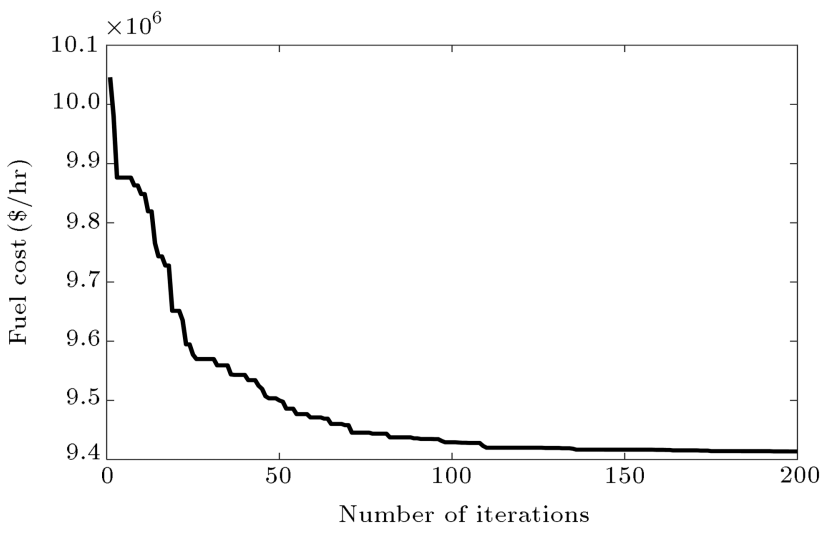

Figure 5. Convergence characteristics of Search Group Optimization (SGO) for 38 generator units.

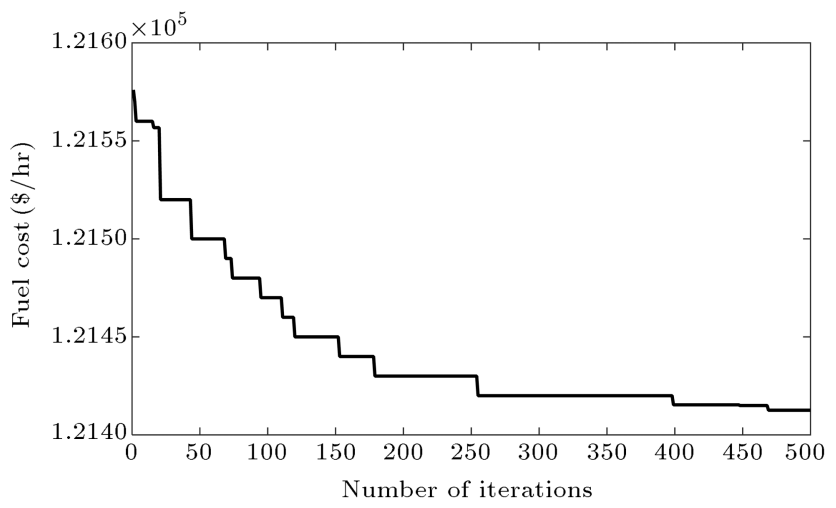

Figure 6. Convergence characteristics of Search Group Optimization (SGO) for 40 generator units. 
Table 8. Minimum, maximum, and average costs obtained by Search Group Optimization (SGO) and various optimization techniques for 38 generator units (50 trials).

\begin{tabular}{|c|c|c|c|c|c|}
\hline \multirow{2}{*}{ Methods } & \multicolumn{3}{|c|}{ Generation cost $(\$ / \mathrm{hr})$} & \multirow{2}{*}{$\begin{array}{c}\text { Time/iteration } \\
\text { (s) }\end{array}$} & \multirow{2}{*}{$\begin{array}{l}\text { No. of hits to } \\
\text { minimum solution }\end{array}$} \\
\hline & Maximum & Minimum & Average & & \\
\hline SGO & 9412259.2481 & 9412256.3019 & 9412256.7730 & 0.15 & 42 \\
\hline RCCRO $[45]$ & 9412411.2099 & 9412404.2774 & 9412407.6050 & 0.29 & 26 \\
\hline $\mathrm{DE} / \mathrm{BBO}[45]$ & 9417245.8795 & 9417235.7863 & 9417241.6400 & 0.65 & 21 \\
\hline AGWO [38] & 9417231.00 & 9417226.00 & 9417229.00 & NA & NA \\
\hline
\end{tabular}

Table 9. Comparison between Search Group Optimization (SGO) and other techniques for the 40-unit test system in terms of optimum power output and fuel cost.

\begin{tabular}{|c|c|c|c|c|}
\hline \multirow{2}{*}{ Unit } & \multicolumn{4}{|c|}{ Power output (MW) } \\
\hline & SGO & EMA $[46]$ & QPSO [46] & IODPSO-G $[46]$ \\
\hline $\mathrm{P} 1$ & 110.7998 & 110.7998 & 111.2000 & 110.8100 \\
\hline $\mathrm{P} 2$ & 110.7998 & 110.7998 & 111.7000 & 110.8000 \\
\hline P3 & 97.3999 & 97.3999 & 97.4000 & 97.4000 \\
\hline $\mathrm{P} 4$ & 179.7331 & 179.7331 & 179.7300 & 179.7300 \\
\hline $\mathrm{P} 5$ & 87.7998 & 87.7999 & 90.1400 & 92.6800 \\
\hline P6 & 139.9999 & 140.0000 & 140.0000 & 140.0000 \\
\hline $\mathrm{P} 7$ & 259.5996 & 259.5996 & 259.6000 & 259.6000 \\
\hline P8 & 284.5996 & 284.5996 & 284.8000 & 284.6000 \\
\hline P9 & 284.5996 & 284.5996 & 284.8400 & 284.6000 \\
\hline $\mathrm{P} 10$ & 130.0000 & 130.0000 & 130.0000 & 130.0000 \\
\hline P11 & 94.0000 & 94.0000 & 168.8000 & 168.8000 \\
\hline $\mathrm{P} 12$ & 94.0000 & 94.0000 & 168.8000 & 168.0000 \\
\hline P13 & 214.7597 & 214.7598 & 214.7600 & 214.7600 \\
\hline $\mathrm{P} 14$ & 394.2793 & 394.2793 & 304.5300 & 304.5200 \\
\hline $\mathrm{P} 15$ & 394.2793 & 394.2793 & 394.2800 & 394.2800 \\
\hline P16 & 394.2793 & 394.2793 & 394.2800 & 394.2800 \\
\hline $\mathrm{P} 17$ & 489.2793 & 489.2793 & 489.2800 & 489.2800 \\
\hline P18 & 489.2793 & 489.2793 & 489.2800 & 489.2800 \\
\hline P19 & 511.2793 & 511.2793 & 511.2800 & 511.2800 \\
\hline P20 & 511.2794 & 511.2793 & 511.2800 & 511.2800 \\
\hline $\mathrm{P} 21$ & 523.2793 & 523.2793 & 523.2800 & 523.2800 \\
\hline $\mathrm{P} 22$ & 523.2793 & 523.2793 & 523.2800 & 523.2800 \\
\hline P23 & 523.2793 & 523.2793 & 523.2900 & 523.2800 \\
\hline P24 & 523.2793 & 523.2793 & 523.2800 & 523.2800 \\
\hline P25 & 523.2793 & 523.2793 & 523.2900 & 523.2800 \\
\hline $\mathrm{P} 26$ & 523.2793 & 523.2793 & 523.2800 & 523.2800 \\
\hline P27 & 10.0000 & 10.0000 & 10.0100 & 10.0000 \\
\hline P28 & 10.0000 & 10.0000 & 10.0100 & 10.0000 \\
\hline P29 & 10.0000 & 10.0000 & 10.0000 & 10.0000 \\
\hline P30 & 87.7999 & 87.7999 & 88.4700 & 87.8400 \\
\hline P31 & 189.9999 & 190.0000 & 190.0000 & 190.0000 \\
\hline P32 & 189.9999 & 190.0000 & 190.0000 & 190.0000 \\
\hline
\end{tabular}


Table 9. Comparison between Search Group Optimization (SGO) and other techniques for the 40-unit test system in terms of optimum power output and fuel cost (continued).

\begin{tabular}{crccc}
\hline \multirow{2}{*}{ Unit } & \multicolumn{4}{c}{ Power output (MW) } \\
\cline { 2 - 5 } & SGO & EMA [46] & QPSO [46] & IODPSO-G [46] \\
\hline P33 & 190.0000 & 190.0000 & 190.0000 & 190.0000 \\
P34 & 164.7998 & 164.7998 & 164.9100 & 164.8000 \\
P35 & 199.9999 & 200.000 & 165.3600 & 164.8100 \\
P36 & 194.3976 & 194.3977 & 167.1900 & 164.8000 \\
P37 & 109.9999 & 110.0000 & 110.0000 & 110.0000 \\
P38 & 109.9999 & 110.0000 & 107.0100 & 110.0000 \\
P39 & 109.9999 & 110.0000 & 110.0000 & 110.0000 \\
P40 & 511.2794 & 511.2793 & 511.3600 & 511.2800 \\
Fuel cost $(\$ / \mathrm{hr})$ & $\mathbf{1 2 1 4 1 2 . 5 3 4 7}$ & 121412.5355 & 121448.2100 & 121414.9300 \\
\hline
\end{tabular}

Table 10. Minimum, maximum, and average costs obtained by Search Group Optimization (SGO) and various optimization techniques for 40 generator units ( 50 trials).

\begin{tabular}{cccccc}
\hline \multirow{2}{*}{ Methods } & \multicolumn{3}{c}{ Generation cost $(\$ / \mathbf{h r})$} & \multirow{2}{*}{$\begin{array}{c}\text { Time/iteration } \\
\text { (S) }\end{array}$} & $\begin{array}{c}\text { No. of hits to } \\
\text { minimum solution }\end{array}$ \\
\cline { 2 - 4 } Maximum & Minimum & Average & $\mathbf{0 . 1 5}$ & $\mathbf{4 0}$ \\
EGO & $\mathbf{1 2 1 4 1 5 . 2 5 8 4}$ & $\mathbf{1 2 1 4 1 2 . 5 3 4 7}$ & $\mathbf{1 2 1 4 1 3 . 0 7 9 4}$ & 0.29 & 21 \\
QPSO [46] & 121416.2031 & 121412.5355 & 121414.6617 & 0.65 & 15 \\
\hline
\end{tabular}

these parameters give different fuel costs. For a single value of one parameter, other parameters must vary for all possible combinations. The tuning of parameters is necessary in order to get an optimized value of the parameters of the SGO algorithm. Herein, different values of $\alpha$ were used in order to get the values of $\varepsilon$ and $t$. Of note, when the value of $\alpha$ is reduced, the subsequent value of $\varepsilon$ also decreases. However, this decrease is not linear as the value of $\varepsilon$ becomes negative after a few trials. These trials of parameter tuning help reach an ideal value for all the parameters. The summarized results of the 40-generator system are shown in Table 11.

Also, using either a large number of search agents or too few search agents for screening the search space does not give the optimized solution. Therefore, a specific number of search agents will only help obtain an optimized solution. For each number of search agents, trials were run. Out of these trials, 50 search agents achieved the optimized fuel cost. For other search agents, no significant improvement in the fuel cost was observed. Moreover, the simulation time duration also lengthened when the number of search agents exceeded 50 . The best output obtained by SGO for each number of search agents in the 40-generator system is given in Table 12 .
Table 11. Effect of various parameters on the performance of Search Group Optimization (SCA).

\begin{tabular}{cccc}
\hline $\boldsymbol{\alpha}$ & $\boldsymbol{\varepsilon}$ & $\boldsymbol{t}$ & Fuel cost $(\$ / \mathbf{h r})$ \\
\hline 2.21 & 0.45 & 1 & 121422.4187 \\
1.97 & 0.39 & 2 & 121419.5698 \\
1.75 & 0.25 & 2 & 121417.1023 \\
1.65 & 0.19 & 1 & 121414.5478 \\
1.31 & 0.15 & 3 & 121413.2013 \\
$\mathbf{1 . 2 7}$ & $\mathbf{0 . 1 2}$ & $\mathbf{3}$ & $\mathbf{1 2 1 4 1 2 . 5 3 4 7}$ \\
1.01 & 0.01 & 2 & 121413.1598 \\
0.71 & -0.09 & 1 & 121415.7541 \\
0.42 & -0.19 & 3 & 121418.2031 \\
0.10 & -0.32 & 2 & 121421.0147 \\
\hline
\end{tabular}

\subsection{Comparative study}

Quality of solution. Tables 1, 3, 5, and 7 illustrate that the fuel cost obtained by the SGO is the least among the other optimization techniques. The cost obtained by SGO was better than that obtained by the many other previously developed algorithms. For example, in Test Case 4, the minimum fuel cost using 
Table 12. Effect of number of search agents on the 40-generator system.

\begin{tabular}{cccccc}
\hline $\begin{array}{c}\text { Number of } \\
\text { search } \\
\text { agents }\end{array}$ & $\begin{array}{c}\text { No. of } \\
\text { hits to } \\
\text { best solution }\end{array}$ & $\begin{array}{c}\text { Simulation } \\
\text { time }(\mathbf{s})\end{array}$ & $\begin{array}{c}\text { Max. } \\
\text { cost } \mathbf{( \$ / h r})\end{array}$ & $\begin{array}{c}\text { Min. } \\
\text { cost } \mathbf{( \$ / h r )}\end{array}$ & $\begin{array}{c}\text { Average } \\
\text { cost (\$/hr) }\end{array}$ \\
\hline 20 & 31 & 47.02 & 121416.2031 & 121413.5847 & 121414.5797 \\
$\mathbf{5 0}$ & $\mathbf{4 4}$ & $\mathbf{5 0 . 4 7}$ & $\mathbf{1 2 1 4 1 5 . 2 5 8 4}$ & $\mathbf{1 2 1 4 1 2 . 5 3 4 7}$ & $\mathbf{1 2 1 4 1 2 . 8 6 1 5}$ \\
100 & 26 & 55.74 & 121417.3647 & 121413.5478 & 121415.3799 \\
150 & 17 & 58.14 & 121419.3201 & 121415.2658 & 121417.9416 \\
200 & 10 & 63.35 & 121422.3795 & 121418.3201 & 121421.5676 \\
\hline
\end{tabular}

the SGO was $121412.5347 \$ /$ hr., which was lower than the minimum cost obtained by using EMA and QPSO. The comparison was made two times, with and without transmission loss. Thus, it is clear that the quality of the solution is the best when SGO is applied.

Robustness. The robustness of any optimization algorithm cannot be judged by only running the algorithm for a single time. A number of trials should be conducted in order to prove the robustness of any optimization technique. According to Tables 2 and 4, SGO achieved the global optimal solution for all the 50 trials in various test cases; in addition, based on Tables 6 and 8, SGO achieved a minimum fuel cost for the maximum number of trials, as compared to other optimization techniques. This proves that the efficiency of the SGO is quite high; therefore, the performance of SGO is superior to other optimization techniques. This proves the robustness of the algorithm.

Computational efficiency. The efficiency of any optimization technique is determined by the time the technique takes to reach the global optimal solution. According to Tables 2, 4, 6, and 8, the computational time taken for one single iteration is the least for the SGO among the other previously developed optimization techniques. Thus, it can be said that SGO gives the global optimal results within the least computational amount of time.

The value of $\alpha$ is $1.27, \varepsilon$ is $0.12, t$ is 3 , and population size is 50 .

\section{Conclusion}

In this paper, a new algorithm called Search Group Optimization Search Group Optimization (SGO) Algorithm was proposed to solve Economic Load Dispatch (ELD) problem. To prove the efficiency of the SGO, four test cases were considered in which the net fuel cost obtained by SGO was compared with that by other optimization techniques in the tabular and graphic forms. The results proved that SGO would be more robust, feasible, and effective than other algorithms in terms of efficiency and computational time. The numerical results also showed that the SGO could prevent premature convergence and had a stable convergence characteristic. Hence, given the exploration and exploitation abilities of the SGO, the problem of ELD was successfully solved.

\section{References}

1. Dhar, R.N. and Mukherjee, P.K. "Reduced-gradient method for economic dispatch", Electrical Engineers, Proceedings of the Institution, 120(5), pp. 608-610 (1973).

2. El-Keib, AA., Ma, H., and Hart, J.L. "Environmentally constrained economic dispatch using the Lagrangian relaxation method", IEEE Trans Power Syst, 9(4), pp. 1723-1729 (1994).

3. Aoki, K. and Satoh, T. "Economic dispatch with network security constraints using parametric quadratic programming", IEEE Power Engineering Review, 2(12), pp. 37-38 (1982).

4. Ching-Tzong, S. and Chien-Tung, L. "New approach with a Hopfield modeling framework to economic dispatch", IEEE Transactions on Power Systems, 15(2), pp. 541-545 (2000).

5. Jabr, R.A., Coonick, A.H., and Cory, B.J. "A homogeneous linear programming algorithm for the security constrained economic dispatch problem", IEEE Transactions on Power Systems, 15(3), pp. 930-936 (2000).

6. Shailti Swamp, K. and Natarajan, A. "Constrained optimization using evolutionary programming for dynamic economic dispatch", Proceedings of 2005 International Conference on Intelligent Sensing and Information Processing, pp. 314-319 (2005).

7. Farooqi, M.R., Jain, P., and Niazi, K.R. "Using Hopfield neural network for economic dispatch of power systems", Proceedings. National Power Engineering Conference, pp. 5-10 (2003). 
8. Sheng-Kuan, W., Chih-Wen, L., and Ji-Pyng, C. "Ant direction hybrid differential evolution for solving economic dispatch of power system", IEEE International Conference on Systems, Man and Cybernetics, 2(1), pp. 1154-1159 (2006).

9. Bavafa, M., Monsef, H., and Navidi, N. "A new hybrid approach for unit commitment using Lagrangian relaxation combined with evolutionary and quadratic programming", Asia-Pacific Power and Energy Engineering Conference, pp. 1-6 (2009).

10. Ali Ahmadi, K., Amir, M., and Naser, S., et al. "Fuzzy economic dispatch and spinning reserve allocation using evolutionary programming", 40th North American Power Symposium, pp. 1-5 (2008).

11. Himanshu, A. and Nitin, N. "Civilized swarm optimization for combined heat and power economic emission dispatch", 7th India International Conference on Power Electronics (IICPE), pp. 1-6 (2016).

12. Yongqiang, W., Jianzhong, Z., and Wen, X., et al. "Economic load dispatch of hydroelectric plant using a hybrid particle swarm optimization combined simulation annealing algorithm", Second WRI Global Congress on Intelligent Systems, 2(1), pp. 231-234 (2010).

13. Chaturvedi, K.T., Pandit, M., and Srivastava, L. "Particle swarm optimization with crazy particles for nonconvex economic dispatch", Appl Soft Comput, 9(1), pp. 962-969 (2009).

14. Lu, H., Sriyanyong, P., and Song, Y.H., et al. "Experimental study of a new hybrid PSO with mutation for economic dispatch with non smooth cost function", Int J Electr Power Energy Syst, 32(1), pp. 921-935 (2010).

15. Taher, N., Farank, G., and Bahman, B. "Modified adaptive PSO algorithm to solve dynamic economic dispatch", IEEE Power Engineering and Automation Conference, 1(1), pp. 108-111 (2011).

16. Jamal, S.A., and Jan, K.S. "Solving economic dispatch problem using hybrid GA-PS-SQP method", IEEE EUROCON, pp. 333-338 (2009).

17. King, D.J. and Oezveren Warsono, C.S. "A genetic algorithm based economic dispatch (GAED) with environmental constraint optimisation", Universities, Power Engineering Conference (UPEC), Proceedings of 46th International, pp. 1-6 (2011).

18. Amjady, N. and Nasiri-Rad, H. "Solution of nonconvex and non-smooth economic dispatch by a new adaptive real coded genetic algorithm", Expert Syst Appl, pp. 5239-5245 (2009).

19. Hazra, J. and Sinha, A.K. "Environmental constrained economic dispatch using bacteria foraging optimization", Joint International Conference on Power System Technology and IEEE Power India Conference, pp. 1-6 (2008).

20. Nagur, P.N., Shubham, R., and Jadhav, H.T. "Modified artificial bee colony algorithm for non-convex economic dispatch problems", International Conference on Green Technologies (ICGT), pp. 258-262 (2012).
21. Deblina, M., Sumit, B., and Chandan, K.C. "Multiobjective economic emission load dispatch using modified biogeography based optimization algorithm", IEEE 7th Power India International Conference (PIICON), pp.1-6 (2016).

22. Shaw, B., Mukherjee, V., and Ghoshal, S.P. "Seeker optimisation algorithm: application to the solution of economic load dispatch problems", IET Generation, Transmission \& Distribution, 5(1), pp. 81-91 (2011).

23. Rahmat, N.A., Musirin, I., and Abidin, A.F., et al. "Economic load dispatch with valve-point loading effect by using differential evolution immunized ant colony optimization technique", Australasian Universities Power Engineering Conference (AUPEC), pp. 16 (2014).

24. Khamsawang, S., Pothiya, S., and Boonseng, C. "Distributed tabu search algorithm for solving the economic dispatch problem", IEEE Region 10 Conference TENCON, 3(1), pp. 484-487 (2004).

25. Roy, P.K. and Mandal, D. "Quasi-oppositional biogeography-based optimization for multi-objective optimal power flow", Electr Power Compon System, 40, pp. 236-256 (2012).

26. Bhattacharya, A. and Chattopadhyay, P.K. "Oppositional biogeography-based optimization for multiobjective economic emission load dispatch", Annual IEEE India Conference (INDICON), pp. 1-6 (2010)

27. Arul, R., Velusami, S., and Ravi, G. "Solving combined economic emission dispatch problems using selfadaptive differential harmony search algorithm", International Conference on Circuits, Power and Computing Technologies [ICCPCT-2014], pp. 757-762 (2014).

28. Chatterjee, A., Ghoshal, S.P., and Mukherjee, V. "Solution of combined economic and emission dispatch problems of power systems by an opposition-based harmony search algorithm", Int J Electr Power Energy Syst, 39(1), pp. 9-20 (2012).

29. Bratati, G., Bishwajit, D., and Bhattacharya, A. "Solving economic load dispatch problem using hybrid Krill Herd algorithm", International Conference on Energy, Power and Environment: Towards Sustainable Growth (ICEPE) (2015).

30. Bhattacharjee, K., Bhattacharya, A., and Sunita, H. "Teaching learning based optimization for different economic dispatch problems", International Journal of Science and Technology, 21(3), pp. 870-884 (2014).

31. Basu, M. "Teaching-learning-based optimization algorithm for multi-area economic dispatch", Energy, 68(15), pp. 21-28 (2014).

32. Roy, P.K., and Bhui, S. "Multi-objective quasioppositional teaching learning based optimization for economic emission load dispatch problem", International Journal of Electrical Power \& Energy Systems, 53, December, pp. 937-948 (2013).

33. Bhattacharjee, K., Bhattacharya, A., and Sunita, H. "Oppositional real coded chemical reaction optimization for different economic dispatch problems", Int $J$ Electr Power Energy Syst, 55, pp. 378-391 (2014). 
34. Bhattacharjee, K. "Economic dispatch problems using backtracking search optimization", International Journal of Energy Optimization and Engineering, IGIglobal, 7(2), Article-2, April-June, pp. 39-60 (2018).

35. Bhattacharjee, K. and Patel, N. "A comparative study of economic load dispatch using sine cosine algorithm", Scientia Iranica, 27(3), pp. 1467-1480 (2018). DOI: 10.24200/sci.2018.50635.1796

36. Xiangzhu, H., Yunqing, R., and Jida, H. "A novel algorithm for economic load dispatch of power systems", Neuro computing, 171, pp. 1454-1461 (2016).

37. Mohammadian, M., Lorestani, A., and Ardehali, M. "Optimization of single and multi-areas economic dispatch problems based on evolutionary particle swarm optimization algorithm", Energy, 161, pp. 710-724 (2018).

38. Bulbul, S., Pradhan, M., and Roy, P.K., et al. "Opposition-based krill herd algorithm applied to economic load dispatch problem", Ain Shams Engineering Journal, 9(3), pp. 423-440 (2016).

39. Bhattacharjee, K. and Patel, N. "A comparative study of economic load dispatch using sine cosine algorithm", Scientia Iranica, 27(3), pp. 1467-1480 (2018). DOI: 10.24200/sci.2018.50635.1796

40. Yang, H.T., Yang, P.C., and Huang, C.L. "A parallel genetic algorithm approach to solving the unit commitment problem: implementation on the transputer networks", IEEE Trans. Power Syst., 1997, 12(2), pp. 661-668 (2016).

41. Bhattacharjee, K., Bhattacharya, A., and Chattopadhyay Discussion on "A GA-API solution for the economic dispatch of generation in power system operation", IEEE Transactions on Power Systems, 28(1), pp. 570-571 (2013).

42. Quande, Q., Cheng, S., and Xianghua, C., et al, "Solving non-convex/non-smooth economic load dispatch problems via an enhanced particle swarm optimization", Applied Soft Computing, 59, pp. 229-242 (2017).

43. Singh, D. and Dhillon, J. "Ameliorated grey wolf optimization for economic load dispatch problem", Energy (2018). DOI: 10.1016/j.energy.2018.11.034.

44. Gonçalves, M., Lopez, R., and Miguel, L., "Search group algorithm: A new metaheuristic method for the optimization of truss structures", Computers and Structures, 153, pp. 165-184 (2015).

45. Bhattacharjee, K., Bhattacharya, A., and Sunita, H. "Chemical reaction optimisation for different economic dispatch problems", IET Gener. Transm. Distrib, 8(3), pp. 530-541 (2014).

46. Naser, G. and Ebrahim, B. "Exchange market algorithm for economic load dispatch", Electrical Power and Energy Systems, 75, pp. 19-27 (2016).

\section{Biographies}

Kuntal Bhattacharjee received a BE degree from BIET, Suri Private College (Burdwan University) and MTech degree from NIT, Durgapur, India in 2003 and 2005, respectively, all in Electrical Engineering. He is currently studying at the Electrical Engineering Department, Institute of Technology, Nirma University, India. His research interests include power system optimization, ELD, EELD, and hydrothermal applications.

Nitish Patel received his BE degree from Babaria Institute of Technology under Gujarat Technological University and is currently pursuing his MTech in Electrical Power Systems from Institute of Technology, Nirma University, Ahmedabad, India. His research interests include artificial intelligence, power system optimization, economic load dispatch, and hydrothermal applications. 